\title{
The Direction of Research on Active Aging and Healthy Life Expectancy in Japan
}

\author{
Atsuko Tokushige', Daiji Araki², Miyuki Suzuki³, Yukie Iwasaki ${ }^{4}$, Mizuho Ozawa $^{5}$ \\ ${ }^{1}$ Faculty of Nursing, Setsunan University, Hirakata, Japan \\ ${ }^{2}$ School of Nursing Science, Meiji University of Integrative Medicine, Nantan, Japan \\ ${ }^{3}$ School of Nursing, Hyogo University of Health Sciences, Kobe, Japan \\ ${ }^{4}$ School of Nursing, Osaka Prefecture University, Habikino, Japan \\ ${ }^{5}$ Graduate School of Nursing, Kobe City College of Nursing, Kobe, Japan \\ Email: tokushige@nrs.setsunan.ac.jp
}

Received 23 March 2014; revised 5 May 2014; accepted 21 May 2014

Copyright @ 2014 by authors and Scientific Research Publishing Inc. This work is licensed under the Creative Commons Attribution International License (CC BY). http://creativecommons.org/licenses/by/4.0/ (c) (i)

\section{Abstract}

In Japan, $18.1 \%$ of the population known as baby-boomers will become the late-stage elderly in 2025, thereby needing a foundation to support this change. The Japanese Ministry of Health, Labour and Welfare is promoting the development of a regional comprehensive system allowing the elderly to continue living in their familiar surroundings. However, a care shortage is inevitable unless elders are able to age in good health, regardless of the system's level of enhancement. This study aims to review the literature on active aging, clarify trends in clinical operations undertakings and research in Japan, and consider relevant research issues. After combining the search results of "active aging" and "healthy life expectancy," we used a text mining technique to analyze the abstracts of 120 original articles and 213 reviews, commentaries, and features. Eight categories were extracted from the original articles: health statistics, gender, age, etc. From the reviews, commentaries, and features, 16 categories were extracted: orientation, disease, and living, etc. Cerebrovascular disease and osteoporosis were the most common diseases covered in the original articles; there has been a substantial amount of research on "active aging" and "healthy life expectancy" because they can easily lead to being bedridden and to a decrease in QOL. In the reviews, commentaries, and features, lifestyle-related diseases and menopause rather than cerebrovascular disease and osteoporosis, were extracted. The categorical differences found in the original articles may be due to the possibility that Japanese researchers are publishing their research abroad rather than in Japan or they submit research on topics that are guaranteed to be published at home or abroad. Little research has been conducted using the terms, "active aging" and "healthy life expectancy," evidenced by the small number of studies generated. Preparations for 2025 will require an increase in the number of studies from the perspective of "active aging" and "healthy life expectancy." 


\section{Keywords}

\section{Active Aging, Healthy Life Expectancy, Text Mining}

\section{Introduction}

The term "active aging" is defined by the World Health Organization (WHO) [1] as "the process of optimizing opportunities for health, participation and security in order to enhance quality of life as people age.” The word "active" in this concept refers to the continued participation in social, economic, cultural, emotional, and civil matters, and does not refer solely to being physically active, nor does it refer solely to having the capacity to engage in labor. "Active aging" also includes elders after retirement, people with disabilities, and those who require care.

In 2000, the WHO surveyed and reported on 191 countries with the understanding that healthy life expectancy is the period in which an independent life can be lived. The "Healthy Japan 21 National Health Promotion Campaign: 2000 to 2012" [2] consisted of an 'extension of healthy life expectancy" and the final report noted an observable trend in this area [2]. Another indicator of health promotion is that approximately $60 \%$ of survey respondents are reported to have reached a target value or be on an improving trend in achieving healthy life expectancy [2]. Healthy Japan 21 plans to deploy a second campaign by 2022, and efforts to extend healthy life expectancy are continuing.

Increasing the number of people who comprise the healthy aging population and live an independent life without receiving long-term care is an important issue in Japan. In 2025, the baby-boomer generation will become the late-stage elderly, and there needs to be a social system to support the 21.79 million citizens [3], which comprise $18.1 \%$ of the total population. As part of a comprehensive regional care system, the Ministry of Health, Labour and Welfare is promoting the construction of a support and service delivery system to allow people to continue living their lives as they wish in their familiar surroundings, until the end of their lives. Regardless of how much the system is enhanced, a nursing shortage is inevitable unless the elderly are able to grow old healthily.

What can we nurses do so that the elderly can be active? Currently, we are in the 12th year since the WHO published the concept of active aging, and the 14th year since the survey on healthy life expectancy was reported. It is important to ascertain the types of efforts that are being undertaken to promote an understanding of the concepts of active aging and healthy life expectancy that will lead to the identification of research issues needing to be addressed.

The present study therefore aims to search the literature on active aging, clarify the trends in the efforts to cope with aging issues and research in Japan, and consider the necessary research issues.

This study analyzed literature information with the text mining method. Text mining is a method that divides sentences into words and phrases drawing upon natural language processing to analyze the frequency of words and the relationships between words. Text mining is an effective tool for objectively analyzing large-volume text data. There are several previous studies that analyze literature titles to explore the research trends [4]-[6]. This study focuses on abstracts instead of titles in an attempt to conduct in-depth analysis of the content of literature. No analysis on Japanese literature information in the aspect of active aging has been conducted thus far, which is a new attempt of the present paper.

\section{Methods}

\subsection{Data Collection}

A document search was performed for the listed publication years of 2000 to 2013 and the search terms of "active aging" and "healthy life expectancy" using the Web edition of the "Japana Centra Revuo Medicina" [7] which is a database of medical publications originating in Japan. It is a representative medical literature database of Japan that enables to retrieve on the web as many as approximately 7.5 million cases of information on academic articles and medical literature collected from 5600 periodicals in the fields of medicine, dentistry, pharmacy, nursing science and other related academic fields published in Japan. This is a well-established service 
that is used by almost all the universities in the fields of medicine, dentistry and nursing science in Japan.

Four articles were extracted using with the term, "active aging” and 1072 with "healthy life expectancy" (accessed at 8pm on 13 April 2014). Next, the conditions of "original articles" and "excl. conference proceedings" were applied to the extracted papers, narrowing the search to original articles, and to commentaries, reviews, and features.

Ultimately, the search yielded two original articles and one review/commentary/feature using the term, "active aging," and 118 original articles and 212 reviews/commentaries/features using "healthy life expectancy.”

\subsection{Data Analysis Methods}

We combined the search results of "active aging” and "healthy life expectancy," and analyzed 120 original articles and 213 reviews, commentaries, and features, using a text mining technique to analyze the abstracts of all of the articles. IBM SPSS Text Analytics for Surveys 4.0 were used to analyze the data. All of the abstracts were read, and the researchers determined whether the extracted literature was appropriate.

A "frequency basis" was employed to create the categories, with a lower limit of ten times for the frequency of occurrence. All of the abstracts were inspected in order to organize the categories, even those with a low frequency of occurrence.

\section{Results}

\subsection{Content of the Original Articles}

The extracted categories and number of records were, in descending order were health statistics (73), gender (61), age (34), research district (25), long-term care insurance (22), disease (20), exercise (9), and lifestyle (5). Table 1 shows the content included in each category.

\section{Table 1. Categories and content extracted from the abstracts of 120 original articles.}

\begin{tabular}{|c|c|c|}
\hline Category & Number of records & Content included \\
\hline \multirow{6}{*}{ Health statistics } & \multirow{6}{*}{73} & Indicators of healthy life expectancy \\
\hline & & Population \\
\hline & & Life table \\
\hline & & Mortality rate \\
\hline & & Average lifespan \\
\hline & & Average life expectancy \\
\hline \multirow{4}{*}{ Gender } & \multirow{4}{*}{61} & Men \\
\hline & & Women \\
\hline & & Gender differences \\
\hline & & Men and women \\
\hline \multirow{4}{*}{ Age } & \multirow{4}{*}{34} & Late-stage elderly \\
\hline & & 85-years-old \\
\hline & & 65 years and older \\
\hline & & 65-years-old \\
\hline \multirow{5}{*}{ Long-term care insurance } & \multirow{3}{*}{25} & Prefectures \\
\hline & & Region \\
\hline & & Nation \\
\hline & \multirow{2}{*}{22} & Requiring nursing care \\
\hline & & Care prevention \\
\hline \multirow{4}{*}{ Disease } & \multirow{4}{*}{20} & State of requiring nursing care \\
\hline & & Osteoporosis \\
\hline & & Impairment \\
\hline & & Cerebrovascular disease \\
\hline Exercise & 9 & Exercise \\
\hline Lifestyle & 5 & Lifestyle \\
\hline
\end{tabular}


The health statistics category included content on disability-adjusted life expectancy (DALE), weighted disability prevalence (WDP), and other indicators of healthy life expectancy, such as population and, mean lifespan. Gender included men, women, gender differences, and men and women. Age included late-stage elderly, 85years-old, 65 years of age or older, and 65 years old. Research district included region, nation, and prefectural and city governments, and revealed that there are some research investigations that have used national data associated with regional initiatives. Long-term care insurance included requiring long-term care and care prevention. Disease included state of requiring long-term care, osteoporosis, impairment, and cerebrovascular disease.

An association between the categories of exercise and lifestyle was observed using the software's "Category web" function. This function makes it possible to visualize the collocations among the categories. Exercise was strongly associated with Indicators of healthy life expectancy in Health statistics (Figure 1). Lifestyle was strongly associated with men, gender differences, and men and women in gender; district and prefecture and city governments in research area and; indicators of healthy life expectancy in health statistics (Figure 2).

\subsection{Content of Reviews, Commentaries, and Features}

The extracted categories and number of records were, in descending order, orientation (134), disease (111), living (70), health statistics (57), QOL (55), exercise (40), care (31), gender (27), medical care (26), research area (19), musculoskeletal (18), falls (16), fitness (15), evidence (14), concept (12), and intervention 11. Table 2 shows the content that was included in each category.

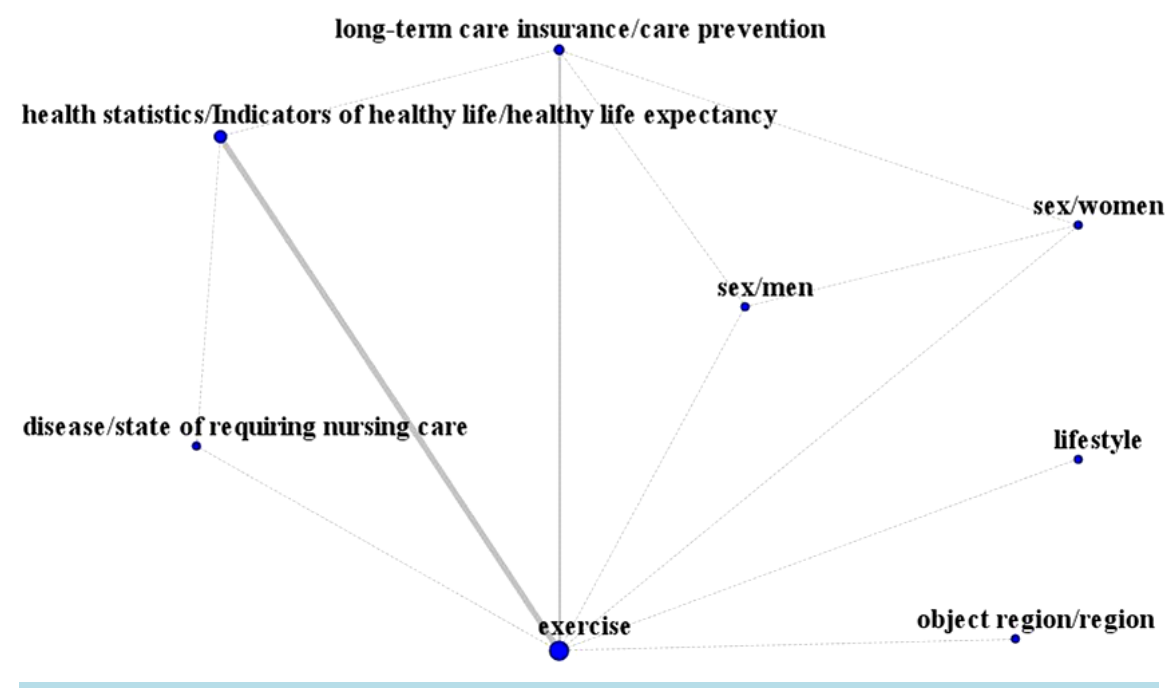

Figure 1. [Exercise] in original manuscript papers and links to each category.

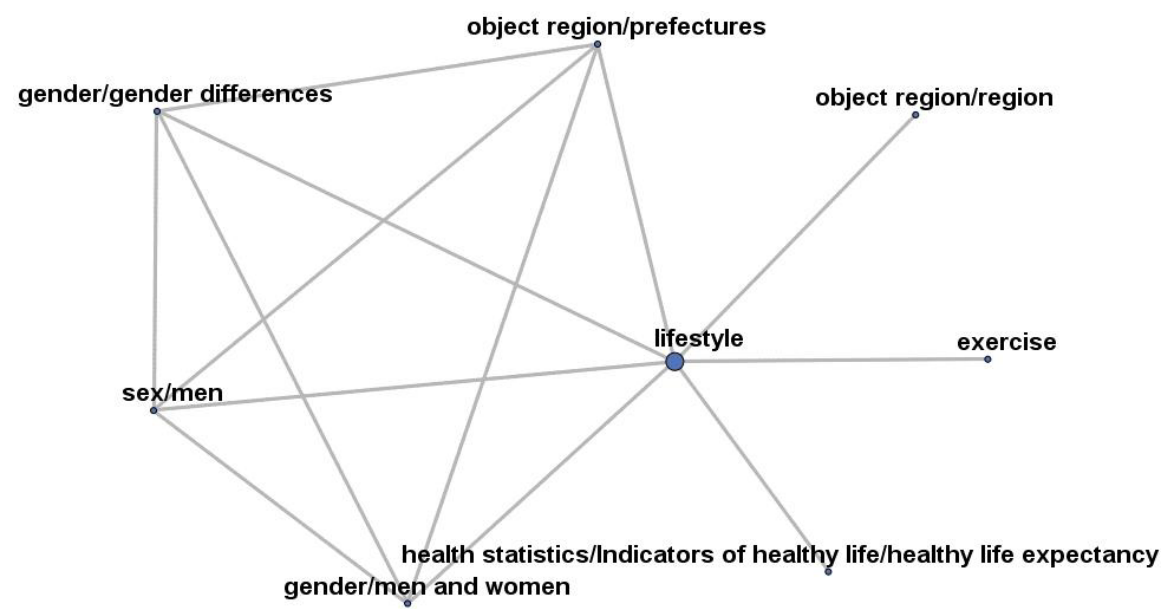

Figure 2. [Lifestyle] in original manuscript papers and links to each category. 
Table 2. Categories and content extracted from the abstracts of 213 reviews, commentaries, and features.

\begin{tabular}{|c|c|c|}
\hline Category & Number of records & Content included \\
\hline \multirow{7}{*}{ Orientation } & \multirow{7}{*}{134} & Prevention \\
\hline & & Extension \\
\hline & & Lengthening \\
\hline & & Maintenance \\
\hline & & Inhibition \\
\hline & & Development \\
\hline & & Independence \\
\hline \multirow{7}{*}{ Disease } & \multirow{7}{*}{111} & Lifestyle-related disease \\
\hline & & Treatment \\
\hline & & Pathology \\
\hline & & Impairment \\
\hline & & Bedridden \\
\hline & & Diagnosis \\
\hline & & Menopause \\
\hline \multirow{3}{*}{ Living } & \multirow{3}{*}{70} & Lifestyle \\
\hline & & Dietary habit \\
\hline & & Diet \\
\hline \multirow{4}{*}{ Health statistics } & \multirow{4}{*}{57} & Lifetime \\
\hline & & Indicator \\
\hline & & Life prognosis \\
\hline & & Longevity \\
\hline \multirow{2}{*}{ QOL } & \multirow{2}{*}{55} & QOL \\
\hline & & Quality \\
\hline Exercise & 40 & Exercise \\
\hline \multirow{3}{*}{ Care } & \multirow{3}{*}{31} & Care prevention \\
\hline & & Requiring nursing care \\
\hline & & Nursing care \\
\hline \multirow{2}{*}{ Gender } & \multirow{2}{*}{27} & Men \\
\hline & & Women \\
\hline Medical care & 26 & Medical care \\
\hline \multirow{2}{*}{ Research area } & \multirow{2}{*}{19} & World \\
\hline & & Nation \\
\hline Musculoskeletal & 18 & Musculoskeletal \\
\hline Falling & 16 & Falling \\
\hline Fitness & 15 & Fitness \\
\hline Evidence & 14 & Evidence \\
\hline Concept & 12 & Concept \\
\hline Intervention & 11 & Intervention \\
\hline
\end{tabular}

Orientation included independence, maintenance, prevention, development, inhibition, lengthening, and extension. Disease included lifestyle-related disease, menopause, diagnosis, bedridden, impairment, pathology, and treatment. Health statistics included lifespan, longevity, life prognosis, and indicators. QOL included QOL and quality; care included care prevention, requiring long-term care, and care; gender included men and women; and research district included world and nation. 
For evidence, intervention, falling, fitness, and medical care, the links between categories were observed using the software's "Category web" function. The results indicated that evidence was strongly associated with life prognosis in health statistics, prevention in orientation, and lifestyle in living. Intervention was most apparently linked to lifestyle in living and to inhibition in orientation (Figure 3). Falls were most strongly linked to prevention in orientation and to musculoskeletal (Figure 4). Fitness was clearly associated with extension in orientation and indicators in health statistics. Medical care was strongly linked to inhibition, prevention, and extension in orientation; lifestyle-related disease in disease; and to care, exercise, living, QOL, and evidence.

\section{Discussion}

\subsection{Content of the Original Articles}

The content reported in the original articles often related to health statistics, and there also was a substantial amount of content relating to gender, because men and women have different average life spans. In terms of the age groups, the early-stage elderly and the late-stage elderly were found to have been participants in research studies.

Osteoporosis and cerebrovascular disease received attention. On September 5, 2013, the Ministry of Health, Labour and Welfare reported that the leading cause of death by age group in Japan was malignant neoplasm for

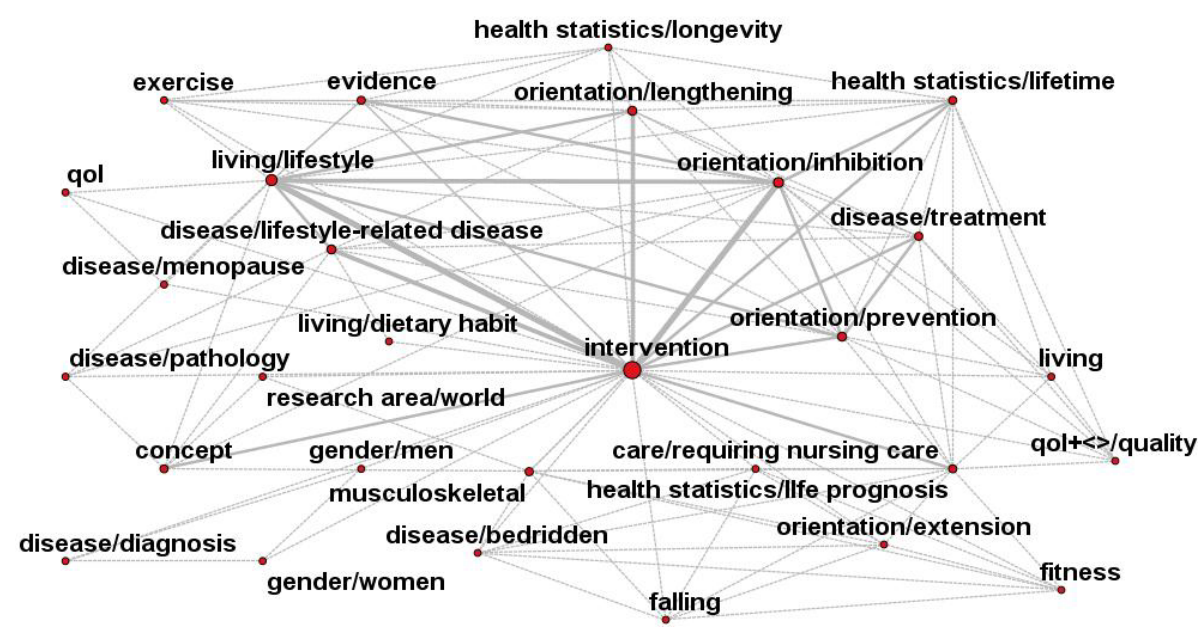

Figure 3. [Intervention] in reviews, commentaries, and features and links to each category.

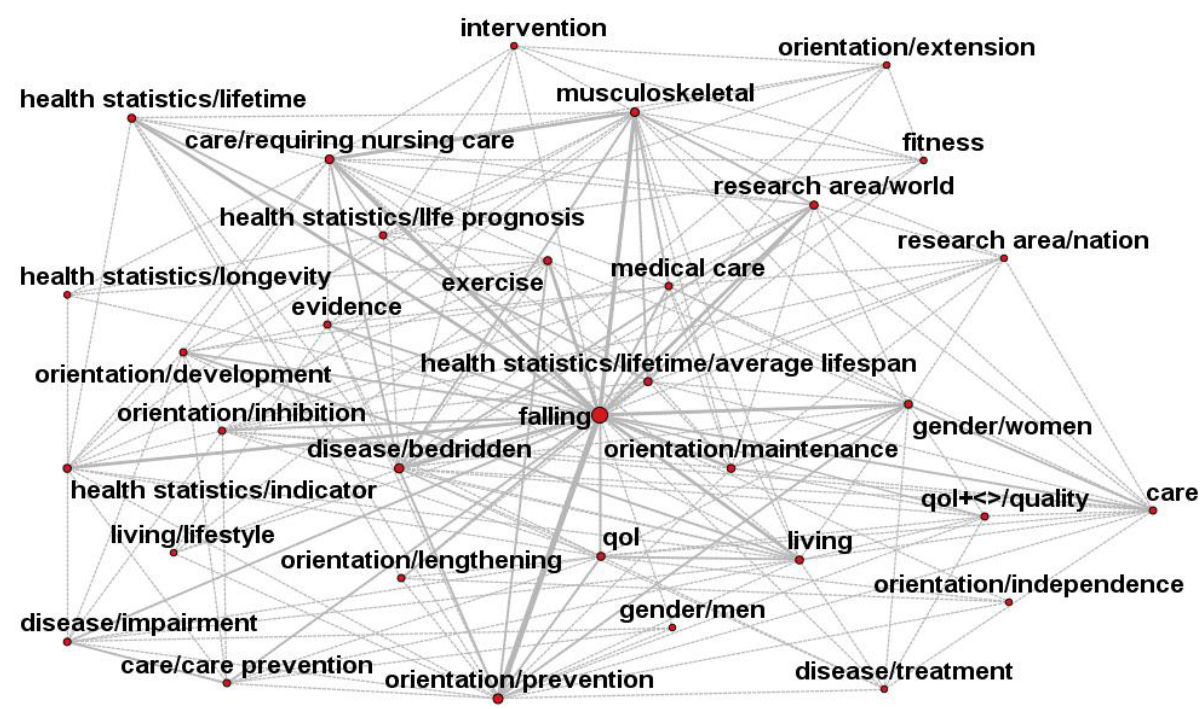

Figure 4. [Falls] in reviews, commentaries, and features and links to each category. 
people ages 65 and older, followed by heart disease in second place, pneumonia in third, and cerebrovascular disease in fourth place. The search that was conducted did not extract malignant neoplasm, heart disease, and pneumonia, which were the leading causes of death at the time of the search. This is thought to occur because treatment is a major component of malignant neoplasm, heart disease, and pneumonia, and therefore they were less likely to be extracted by the techniques used in this study. Cerebrovascular disease and osteoporosis, however, are more likely to lead to being bedridden and to having a decrease in QOL, and this is presumably the reason for the substantial amount of attention to research on "active aging" and "healthy life expectancy." In the case of malignant neoplasms, it has been reported from the fit of the graph of cause-specific mortality to the Gompertz curve, that primary prevention of lifestyle-related diseases is important [8]. This is an issue that should be addressed by future research.

The fact that the "Category web" function showed a strong association between exercise and indicators of healthy life expectancy in health statistics demonstrates that the research on exercise is associated with indicators of healthy life expectancy. In the Japana Centra Revuo Medicina database, the search using "training and healthy life expectancy" extracted three original articles and 26 reviews, commentaries, and features. The "National Survey on Active Aging 2009” [9], performed by the Japan Health Promotion \& Fitness Foundation, reported "time to enjoy exercise and sports as a realistic goal to increase the amount of time spent in these activities that was most desired by the middle-aged and elderly (21.6\%). This finding showed that about one in five middle-aged and elderly adults put into practice, to the goals they thought they would achieve, during the oneyear period. We feel that in the future, research on exercise/training and healthy life expectancy is an important topic that needs to be addressed.

\subsection{Content of the Reviews, Commentaries, and Features}

The extracted category of disease included lifestyle-related diseases, menopause, diagnosis, bedridden, impairment, pathology, and treatment, and did not generate osteoporosis and cerebrovascular disease unlike the original articles. The diseases referred to as lifestyle-related diseases and menopause were extracted. The categories of Orientation, QOL, falls, fitness, evidence, concept, and intervention, which were not generated in search of the original articles, were generated here. The differences in categories from those of the original articles may be attributed to the possibility that Japanese researchers are not publishing their research studies as original articles domestically but rather are publishing them abroad. Another possibility is that Japanese researchers are selecting, and submitting studies on research topics that are almost guaranteed to be published both at home and abroad. In the links between the categories in the "Category web" function, intervention was most strongly linked to lifestyle-related disease in living, and falls was most strongly linked to prevention in orientation as well as exercise; the focus of the research is inferred to be oriented toward intervention in lifestyle and fall prevention and exercise. This finding seems to be a research topic that needs further attention in future studies, as well.

\section{Conclusions}

Using a domestic database search to examine the differences in content between original articles and reviews, commentaries, and features revealed differences in the categories that were extracted. This study found that the research presentations in domestic publications have a different form from reviews, commentaries, and features. For reviews, commentaries, and features, regarding QOL, falls, fitness, and intervention, and other categories not extracted from the original articles, we predict that if the number of domestic publications increases, the number of people who might use those sources will also increase; therefore, an increase in the number of domestic publications is regarded as desirable.

This study also found that research using the term "active aging” is substantially low in Japan. Even the addition of the keyword, "healthy life expectancy" only generated 120 original articles and 213 reviews, commentaries, and features over 13 years. Additionally, searching the database using the keywords long-term care insurance and care generated results that were not substantially better. The category of nursing, also, was generated neither in the original articles nor in the reviews, commentaries, and features, and there was little research relating to active aging and healthy life expectancy conducted by the nursing profession.

In preparing the comprehensive regional care system for 2025, it is essential to remember that the absence of an effective approach to the health of the body and mind as its foundation, will produce a problematic service shortage. Malignant neoplasm, heart disease, and pneumonia, which are the three leading causes of death in Ja- 
pan, failed to be extracted using this study's methods. However, it is apparent that approaches to countering these diseases will extend the healthy life expectancy of Japanese adults. Similarly, it also will be important to have approaches to problems of depression and suicide in the elderly, problems, which were not extracted here. In terms of nursing, performing intervention research on lifestyle or mental support as a form of primary prevention of malignant neoplasm, heart disease, and pneumonia is required.

\section{Limitations of the Study}

The only database used in this study was the "Japana Centra Revuo Medicina (Web edition, Ver. 5)"; therefore, the present study, which was conducted inside Japan, did not provide a full and comprehensive examination of the topic. Similar analyses are deemed necessary in other academic fields than medicine, dentistry, pharmacy, and nursing science. In the future, it will be necessary to use other databases in conjunction with foreign databases to attempt to gain knowledge of the international implications of research trends on active aging. 165 hits were recorded when we searched for "active aging" in the Cochrane Database of Systematic Reviews [10]. We are planning to analyze the contents of these reviews.

\section{References}

[1] Active Ageing: A Policy Framework (2002) World Health Organization, 21-18. http://www.who.int/ageing/active_ageing/en/

[2] Ministry of Health, Labour and Welfare (2012) Healthy Japan 21 Final Assessment, 6-7. http://www.mhlw.go.jp/stf/houdou/2r9852000001r5gc.html

[3] Ministry of Health, Labour and Welfare (2013) On the Future Prospects of the Elderly Population. http://www.mhlw.go.jp/stf/seisakunitsuite/bunya/hukushi_kaigo/kaigo_koureisha/chiiki-houkatsu/

[4] Aoki, M. and Aoki, K. (2009) Analysis of Asbestos Research Conducted in Japan : Analyses of The Thesaurus Found in Japan: Analyses of The Thesaurus Found in The Literature and Free Terms Contained in The Titles. Juntendo Medical Journal, 55, 478-486.

[5] Shiromaru,M., Mizutani, S., Matsumoto, H. (2012) Trends in the Medical Studies on "Stress-Coping” In Japan: A Text-Mining Analysis. Sapporo Journal of Health Sciences, 1, 519-528.

[6] Cho,M.,Taniguchi, T., Harano, K.,et al.(2013) Trends of Study in Care and Welfare in the "Research Journal of Care and Welfare”: Text Mining Using a Paper Title. Research Journal of Care and Welfare, 20, 152-158.

[7] Japana Centra Revuo Medicina, Web Database, Japan Medical Abstracts Society. http://login.jamas.or.jp/enter.html

[8] Suzuki, T. (2010) Relationship between the Concept of Healthy Life Expectancy and Methods of Extending it. In: Orimo, H., Ed., Gerontology, 2nd Edition, University of Tokyo Press, Tokyo, 519-528.

[9] The National Survey on Active Aging 2009 (2010), Japan Health Promotion \& Fitness Foundation, Tokyo, 2-5.

[10] Cochrane Library, Web Database, Cochrane Collaboration. http://www.thecochranelibrary.com/view/0/index.html 